\title{
Serum Ferritin and D-Dimer as Possible Risk Factors in Ischaemic Stroke in Cancer Patients
}

\author{
Mohammed Abbas1, Ahmed Hady², Mohamed Jawad', Mohamed Mofreh ${ }^{3}$, Samer Salama1, \\ Wessam Mustafa ${ }^{1}$
}

\author{
${ }^{1}$ Neurology Department, Faculty of Medicine, Mansoura University, Mansoura, Egypt \\ ${ }^{2}$ Clinical Oncology and Nuclear Medicine Department, Faculty of Medicine, Mansoura University, Mansoura, Egypt \\ ${ }^{3}$ Department of Clinical Pathology, Mansoura University, Mansoura, Egypt \\ Email:mohammedabbas@mans.edu.eg,hadyonc@mans.edu.eg,mohreh@mans.edu.eg,m7md3zt@gmail.com, \\ samer_salama@mans.edu.eg,wesam010@mans.edu.eg
}

How to cite this paper: Abbas, M., Hady, A., Jawad, M., Mofreh, M., Salama, S. and Mustafa, W. (2021) Serum Ferritin and D-Dimer as Possible Risk Factors in Ischaemic Stroke in Cancer Patients. World Journal of Neuroscience, 11, 307-317.

https://doi.org/10.4236/wjns.2021.114022

Received: October 31, 2021

Accepted: November 27, 2021

Published: November 30, 2021

Copyright $\odot 2021$ by author(s) and Scientific Research Publishing Inc. This work is licensed under the Creative Commons Attribution International License (CC BY 4.0).

http://creativecommons.org/licenses/by/4.0/

(c) (i) Open Access

\begin{abstract}
Background: Ischemic stroke is frequently encountered in patients with malignant disease. The pathophysiology of stroke in such cases differs from other subjects with no malignant disease. This study was conducted to compare serum levels of ferritin and d-dimer in cases with ischemic stroke in cancer versus non-cancer patients. Patients and methods: The data of consecutive 264 patients presented with ischemic stroke, confirmed by clinical examination and radiological investigations, were retrospectively reviewed. The included cases were divided into two groups: Group A (non-cancer with stroke, 210 cases) and Group B (cancer with stroke, 54 cases). The collected data included patient demographics, systemic comorbidities, disease and tumor characteristics, in addition to platelet count, serum ferritin and d-dimer. Results: Age, gender, and systemic comorbidities were statistically comparable between the two groups. Additionally, the etiology of stroke and its disability were not statistically different between the two groups. However, the incidence of mortality significantly increased in Group B $(25.93 \%$ vs. $7.14 \%$ of Group $A, p=0.005)$. Both serum ferritin and d-dimer showed a significant increase in association with cancer (Group B). The former had mean values of 294.54 and $867.87 \mathrm{ng} / \mathrm{ml}$, while the latter had mean values of 463.83 and $888.13 \mathrm{ng} / \mathrm{ml}$ in the same two groups, respectively. Conclusion: Serum ferritin and d-dimer showed a significant rise in cancer-associated ischemic stroke. This confirms the role of the hypercoagulable state, associated with malignancy in the development of this morbidity.
\end{abstract}

\section{Keywords}

Ischemic Stroke, Cancer, Non-Cancer, Risk Factors 


\section{Introduction}

Ischemic stroke and cancer share common risk factors for ischemic stroke in cancer patients [1]. Autopsies taken from cancer patients revealed that about 14.6 percent had cerebrovascular pathology, and 7.4 percent had clinical manifestations of stroke, according to a previous report [2].

The etiology of cancer-associated ischemic stroke could greatly differ from others who do not have malignant disease. In patients with malignancy, stroke may occur due to the hypercoagulable state associated with cancer, direct tumor compression, non-bacterial thrombotic endocarditis, or as a complication from anti-cancer therapy [3] [4] [5].

Biomarkers for stroke can be utilized to understand pathogenesis better, screen high-risk individuals, predict clinical outcomes, and treat stroke patients effectively [6] [7]. These biomarkers include C-reactive protein (CRP), brain natriuretic peptide (BNP), and erythrocyte sedimentation rate (ESR) [7] [8].

Coagulopathy-related pathologies can also potentiate ischemic stroke in cancer patients. D-dimer, a by-product of the blood clotting and break-down process, is a direct measure of active coagulation. It has been widely employed as a reliable test of hypercoagulability in numerous earlier investigations [9] [10]. Some studies noted its significant rise in cancer-associated ischemic stroke compared to non-cancer patients [11] [12].

The largest iron reserve in the human body is ferritin, which is one of the acute-phase proteins [13]. Acute-phase response proteins are thought to play a crucial role in the pathological process of ischemic stroke. This was confirmed by previous research, which showed that high ferritin levels are strong predictors for ischemic stroke [14]. More recent research notes significant elevation of the previous markers associated with stroke in lymphoma patients compared to those who did not [15].

This study evaluated serum levels of ferritin and d-dimer in cases with ischemic stroke in cancer patients compared to non-cancer patients.

\section{Patients and Methods}

This retrospective study was conducted at Mansoura University Neurology Department. We retrospectively reviewed the data of ischemic stroke patients presented and admitted to our department between January 2018 and December 2020.

We included patients diagnosed with acute ischemic stroke during the study period. The diagnosis of ischemic stroke was established when a new vascular lesion was detected on brain imaging (computed tomography CT or magnetic resonance imaging MRI) explaining patient clinical manifestations. Contrarily, cases with recurrent stroke, transient ischemic attacks (TIA), negative brain radiology, primary brain tumor or brain metastasis were excluded.

A total of 264 cases were included in the current study, of whom 210 patients had a stroke without cancer (Group A), and the remaining 54 patients had a 
stroke in association with cancer (Group B).

Regarding the ethical consideration, it gained approval from the local ethical committee and Institutional Review Board (IRB) of Mansoura University. Additionally, informed written consent was signed by all participants (or their relatives in case of disturbed conscious level) after an explanation of the benefits and possible complications of each intervention performed.

The patient evaluation included detailed history taking, thorough clinical examination and routine laboratory investigations. Patient history included personal history, current complaint with its analysis, and detailed medical history regarding diabetes, hypertension, ischemic heart disease, cardiac dysrhythmia, or previous stroke events. Cancer-related data included the primary tumor site, duration of disease, and the presence of disseminated disease. Full neurological assessment was done for all cases, and the National Institutes of Health Stroke Scale (NIHSS) [16] was calculated for all subjects at admission.

The radiological investigation included brain CT and/or MRI was ordered to confirm the diagnosis. This was ordered for all patients. Other radiological investigations included transthoracic echocardiography, carotid doppler study, and transcranial doppler, and they were ordered in most of the cases to determine stroke etiology. The etiology of stroke was classified according to the Trial of Org 10172 in Acute Stroke Treatment (TOAST) classification criteria [17].

Laboratory investigations included platelet count, serum ferritin and d-dimer levels. Serum ferritin and dimer concentrations were measured using the classic ELISA technique. The former was measured by AccuBind serum ferritin kit (Monobind Inc., USA), while the latter was assessed by ZYMUTEST D-Dimer ARK023A kits (Hyphen Biomed, France).

The previous data were collected to be analyzed and compared between cancer and non-cancer patients. Our primary objective was to compare ferritin and $\mathrm{d}$ dimer levels between the two groups. Other objectives included the difference in clinical characteristics and mortality between the two groups.

To analyze the data, we used the SPSS 26 statistical analysis package from IBM/SPSS Inc. in Chicago, IL. Patient baseline characteristics were presented as either frequencies and percentages (\%) or mean and standard deviations (SD). To compare data, we applied the Chi-Square test (or Fisher's exact test) to compare two independent groups of qualitative data, whereas we used the Mann-Whitney U test and independent-Samples t-test to compare two groups of non-parametric and parametric quantitative data, respectively. A p-value less than 0.05 was considered statistically significant.

\section{Results}

As shown in Table 1, patient demographics, including age and gender, were statistically comparable between the two groups $(\mathrm{p}>0.05)$. The prevalence of smoking was $41.9 \%$ and $37.04 \%$ in Groups A and B, respectively. Regarding systemic comorbidities, it was more or less similar between the two groups, with no 
Table 1. Demographic and clinical history data of the two study groups.

\begin{tabular}{|c|c|c|c|}
\hline & Group A $(n=210)$ & Group B $(n=54)$ & $\mathrm{p}$ value \\
\hline Age (years) & 66.289 .83 & $64.08 \pm 8.89$ & 0.447 \\
\hline \multicolumn{4}{|l|}{ Gender } \\
\hline -Male & $143(68.10 \%)$ & $38(70.73 \%)$ & \multirow[t]{2}{*}{0.562} \\
\hline -Female & $67(31.90 \%)$ & $16(29.63 \%)$ & \\
\hline Smoking & $88(41.90 \%)$ & $20(37.04 \%)$ & 0.257 \\
\hline \multicolumn{4}{|l|}{ Medical history } \\
\hline -Hypertension & $149(70.95 \%)$ & $34(62.96 \%)$ & 0.126 \\
\hline -Diabetes mellitus & $85(40.48 \%)$ & $25(46.30 \%)$ & 0.158 \\
\hline -Atrial fibrillation & $59(28.10 \%)$ & $13(24.10 \%)$ & 0.214 \\
\hline -Dyslipidemia & $37(17.62 \%)$ & $7(12.96 \%)$ & 0.178 \\
\hline -Coronary artery disease & $22(10.48 \%)$ & $8(12.50 \%)$ & 0.382 \\
\hline \multicolumn{4}{|l|}{ TOAST subtype } \\
\hline -Large vessel disease & $111(52.86 \%)$ & $25(46.30 \%)$ & \multirow{5}{*}{0.288} \\
\hline -Cardioembolism & $42(20 \%)$ & $12(22.22 \%)$ & \\
\hline -Small vessel disease & $25(11.90 \%)$ & $8(14.81 \%)$ & \\
\hline -Other determined cause & $5(2.38 \%)$ & $2(3.7 \%)$ & \\
\hline -Not determined & $27(12.86 \%)$ & $7(12.96 \%)$ & \\
\hline NIHSS at admission & $14.35 \pm 3.51$ & $14.25 \pm 4.02$ & 0.260 \\
\hline In-hospital mortality & $15(7.14 \%)$ & $14(25.93 \%)$ & $0.005^{*}$ \\
\hline
\end{tabular}

NIHSS: National Institutes of Health Stroke Scale TOAST: Trial of Org 10,172 in Acute Stroke Treatment.

significant difference detected. Hypertension was the most common comorbidity in the two groups, followed by diabetes mellitus. Other comorbidities included atrial fibrillation, dyslipidemia and coronary artery disease.

NIHSS had mean values of 14.35 and 14.25 in the two study groups, respectively $(\mathrm{p}=0.260$ ). Additionally, the etiology of stroke was also comparable between the two groups according to the TOAST classification $(\mathrm{p}=0.288)$. In-hospital mortality was encountered in 15 (7.14\%) and 14 (25.93\%) patients in the same groups, respectively, with a significant increase in association with malignancy.

When it comes to laboratory parameters, platelet count was statistically comparable between the two groups ( $\mathrm{p}=0.132$ ), which had mean values of 306 and $324 \times 10^{3} / \mathrm{ml}$ in groups A and B, respectively. On the other hand, both serum ferritin and dimer showed a significant increase in association with cancer (Group B) ( $<$ 0.001). The former had mean values of 294.54 and $867.87 \mathrm{ng} / \mathrm{ml}$, while the latter had mean values of 463.83 and $888.13 \mathrm{ng} / \mathrm{ml}$ in the same two groups, respectively. The previous data are shown in Table 2 .

In Group B, primary cancer sites were distributed as follows; gastrointestinal 
Table 2. Laboratory parameters in the two study groups.

\begin{tabular}{cccc}
\hline & Group A (n=210) & Group B (n=54) & p value \\
\hline Platelet count $\times 10^{3} / \mathrm{ml}$ & $306(120-470)$ & $324(110-550)$ & 0.132 \\
D-dimer $(\mathrm{ng} / \mathrm{ml})$ & $463.83 \pm 153.07$ & $888.13 \pm 256.09$ & $<0.001^{\star}$ \\
Ferritin $(\mathrm{ng} / \mathrm{ml})$ & $294.54 \pm 113.53$ & $867.87 \pm 291.93$ & $<0.001^{\star}$ \\
\hline
\end{tabular}

Table 3. Clinical data in cancer patients with stroke (Group B).

\begin{tabular}{lc}
\hline \multicolumn{1}{c}{ Item } & $(\mathbf{n}=\mathbf{5 4})$ \\
\hline Type of primary cancer & \\
-GIT & $23(42.59 \%)$ \\
-Breast & $13(24.07 \%)$ \\
-Lung & $9(16.67 \%)$ \\
-Urogenital & $6(11.11 \%)$ \\
-Hematological & $3(5.56 \%)$ \\
Duration of cancer (year) & $3(2-5)$ \\
Presence of metastasis & $23(42.59 \%)$ \\
\hline
\end{tabular}

tract (42.59\%), breast (24.07\%), lung (16.67\%), urogenital (11.11\%), and hematological (5.56\%). Distant metastasis was detected in 23 patients (42.59\%). The mean duration of malignancy ranged between two and five years ( median $=3$ ). Table 3 summarizes these data.

\section{Discussion}

Cerebrovascular disease and cancer are two of the most common diseases that contribute to death or disability around the world. The risk of an ischemic stroke in patients with malignant disease is substantially higher than in the general population, as has been documented in the literature [18] [19] [20]. This should encourage us to increase the study into the underlying causes and mechanisms of cancer-associated ischemic stroke.

This study was conducted to evaluate serum levels of ferritin and d-dimer in cases with ischemic stroke in cancer patients compared to non-cancer patients. We reviewed the data of consecutive 264 patients, who were divided into two groups; Group A (210 non-cancer patients with stroke) and Group B (54 cancer patients with stroke).

According to the previously mentioned results, we did not detect any significant difference between the two study groups in terms of patient demographics and most of their clinical risk factors and should nullify any bias that might have skewed the results in favor of one group rather than the other one.

In the current study, current smoking was reported by $41.9 \%$ and $37.04 \%$ of patients in the two groups, respectively (A and B), with no statistical difference detected $(p=0.257)$. Likewise, other authors denied any significant difference 
between cancer and non-cancer groups regarding the prevalence of smoking ( $\mathrm{p}$ $=0.40$ ), which was reported by $17.9 \%$ and $21.8 \%$ of patients in the two groups, respectively [1]. Smoking is a significant risk factor for malignancy and ischemic stroke, and it is also the underlying etiology of the vast majority of ischemic strokes in cancer patients [21] [22].

Our findings showed no difference between the study groups regarding the prevalence of hypertension $(\mathrm{p}=0.126)$. It was detected in $70.95 \%$ and $62.96 \%$ of patients in groups A and B, respectively. Romeiro and his associates reported that hypertension was present in $71.43 \%$ and $79.47 \%$ of patients in cancer and non-cancer groups, respectively, with no statistical difference detected $(p>0.05)$ [23]. On the other hand, another study reported a higher prevalence of hypertension in the non-cancer group ( $78.8 \%$ compared to $54.5 \%$ in the cancer group $\mathrm{p}<0.01)[1]$.

In the current study, diabetes mellitus was present in $40.48 \%$ and $46.3 \%$ of patients in Groups A and B, respectively $(p=0.158)$. Stefan and his associates also reported no significant difference between the two groups regarding the prevalence of diabetes ( $p>0.05$ ), which was $24 \%$ and $28 \%$ of patients in cancer and non-cancer groups, respectively [24]. Other authors confirmed the previous findings [25]. In contrast to the previous findings, another study reported a higher prevalence of diabetes in the non-cancer group (33.77\% compared to only $16.07 \%$ of patients in the cancer group, $\mathrm{p}<0.05)$ [23].

In our study, atrial fibrillation was detected in $28.1 \%$ and $24.01 \%$ of patients in groups A and B, respectively, which was statistically insignificant between the two study groups $(\mathrm{p}=0.214)$. In another study, authors reported that atrial fibrillation was encountered in 15.25 and $20.6 \%$ of patients in cancer and non-cancer groups, respectively, with no statistical difference between the two groups $(p=0.382)$ [26]. Contrarily, Kim and Lee reported a higher prevalence of the same pathology in the non-cancer group $(\mathrm{p}<0.01)$, as it was present in $23.1 \%$ of cases in that group, compared to only $9.6 \%$ in the cancer group [1].

In the current study, the prevalence of dyslipidemia did not significantly differ between the two groups ( $\mathrm{p}=0.178$ ), as it was present in $17.62 \%$ and $12.96 \%$ of patients Groups A and B, respectively. Romeiro et al. also reported a comparable prevalence of dyslipidemia between cancer and non-cancer groups ( $p>0.05)$. Nevertheless, the reported incidence was higher than ours, as this morbidity was detected in $39.29 \%$ and $52.89 \%$ of patients in cancer and non-cancer groups, respectively [23]. However, other authors reported a significant difference between the same two groups regarding dyslipidemia $(\mathrm{p}<0.01)$, which was present in $6.4 \%$ and $39.7 \%$ of patients in cancer and non-cancer groups, respectively [1].

Our findings showed that coronary artery disease was present in $10.48 \%$ and $12.5 \%$ of patients in Groups A and B, respectively, which yielded no significant difference between the two groups on statistical analysis. In line with our findings, Sorgun et al. reported that the same cardiac morbidity was noted in $21.7 \%$ and $23.4 \%$ of patients in cancer and non-cancer groups, respectively $(p=0.799)$ 
[26]. Other authors confirmed the previous findings [24].

In the current study, the etiology of stroke was also comparable between the two groups according to the TOAST classification $(p=0.288)$. Likewise, Bang and his associates reported similar stroke subtypes between cancer and non-cancer patients [13]. Moreover, Zhang et al. confirmed the previous findings as TOAST classification was not statistically significant between cancer and non-cancer stroke patients [27]. However, in another study, cancer patients with stroke showed a higher incidence of large-artery atherosclerosis and undetermined etiology and lower incidence of small artery occlusion and cardioembolism when compared to non-cancer patients $(p=0.02)$ [1]. The difference in stroke etiology between different studies could be explained by different sample sizes and patient characteristics.

In our study, platelet count was statistically comparable between the two groups ( $\mathrm{p}=0.132$ ), and this is in agreement with Sorgun and his colleagues, who reported that the same parameter was statistically comparable between the two groups (218 vs. $229.5 \times 10^{3} / \mathrm{ml}$ in cancer and non-cancer groups respectively) [26].

Our findings showed significant elevation of serum d dimer levels in association with cancer. It had mean values of 463.83 and $888.13 \mathrm{ng} / \mathrm{ml}$ in groups $\mathrm{A}$ and $B$, respectively $(\mathrm{p}<0.001)$. A previous study also confirmed the previous findings, as the same parameter had mean values of 2370.5 and $324.2 \mathrm{ng} / \mathrm{ml}$ in cancer and non-cancer groups, respectively $(\mathrm{p}<0.001)[1]$.

Sorgun et al. reported that d dimer had median values of 1519 and $590 \mathrm{ng} / \mathrm{ml}$ in cancer and non-cancer groups, respectively, with a significant rise in cancer patients $(\mathrm{p}<0.001)$ [26]. An additional study confirmed the previous findings [28].

Multiple studies have incriminated both hypercoagulability and embolism in the development of ischemic stroke in cancer patients. Cancer patients with ischemic stroke (who had no other common stroke risk factors) had significantly higher levels of $d$ dimer that corresponded with the rise in embolic signal during transcranial Doppler monitoring. [11] [13] [29].

When it comes to serum ferritin in the current study, it showed a significant increase in association with cancer $(\mathrm{p}<0.001)$. It had mean values of 294.54 and $867.87 \mathrm{ng} / \mathrm{ml}$ in Groups A and B, respectively. To the best of our knowledge, there is a clear paucity of studies handling the role of serum ferritin in cancer-associated ischemic stroke. This represents a point of strength in favor of our study.

Wei and his coworkers also reported that high ferritin level was a strong predictor for ischemic stroke development in patients with non-Hodgkin lympho$\mathrm{ma}(\mathrm{p}<0.001)$. It had mean values of $564 \mathrm{ng} / \mathrm{ml}$ in cases that developed stroke, compared to $323.41 \mathrm{ng} / \mathrm{ml}$ in cases that did not develop that complication [15]. In cancer patients, elevated serum ferritin, a protein functioning as iron storage, is associated with inflammation and is also linked to a hypercoagulable condition [30] [31] [32]. 
Serum ferritin's precise mechanism in a hypercoagulable condition is yet unknown, but we hypothesized that it could cause a significant inflammatory response, resulting in hypercoagulability, which in turn enhances the development of ischemic stroke in patients with malignant neoplasms.

Gastrointestinal tract cancer was the commonest one encountered in our series $(42.59 \%)$. This was followed by breast $(24.07 \%)$, lung (16.67\%), urogenital (11.11\%), and hematological (5.56\%). Another study reported gastric cancer was the commonest primary tumor in the included cancer population with stroke (21.2\%), followed by lung cancer (16\%), colorectal cancer (14.1\%) and hepatobiliary cancer (12.8\%). Other malignancies included prostate, breast, pancreas, cervical, urological and hematological neoplasms [1].

To date, a number of studies have sought to determine which types of cancer are most closely associated with stroke [33]. According to a previous study, among the included 1274 stroke patients, $13 \%$ of cases were also diagnosed with cancer, with the most common cancer kinds being urogenital, breast, and gastrointestinal [24]. Furthermore, a greater risk of stroke was observed in cancer patients who had been diagnosed with lung, pancreatic, colorectal, breast, or prostate cancer [34].

Our findings showed a significant increase in mortality rate in association with cancer ( $p=0.005)$, which was encountered in $25.93 \%$ of cancer patients, compared to $7.14 \%$ of non-cancer cases. Similarly, other authors reported that the presence of cancer was associated with a significant rise in in-hospital mortality in patients with stroke $(\mathrm{p}=0.013)$. Mortality occurred in $21.7 \%$ and $9.9 \%$ of cases in cancer and non-cancer groups, respectively [26].

Our study has some limitations. It was conducted in a single center in nature, and the relation between the two study's biomarkers and patient prognosis should have been evaluated. These cons should be handled in the upcoming studies.

\section{Conclusion}

Based on the previous findings, serum ferritin and d-dimer showed a significant increase in cancer-associated ischemic stroke. This confirms the role of the hypercoagulable state, associated with malignancy in the development of this morbidity.

\section{Conflicts of Interest}

The authors declare no conflicts of interest regarding the publication of this paper.

\section{References}

[1] Kim, K. and Lee, J.-H. (2014) Risk Factors and Biomarkers of Ischemic Stroke in Cancer Patients. Journal of Stroke, 16, 91-96. https://doi.org/10.5853/jos.2014.16.2.91 
[2] Graus, F., Rogers, L.R. and Posner, J.B. (1985) Cerebrovascular Complications in Patients with Cancer. Medicine, 64, 16-35. https://doi.org/10.1097/00005792-198501000-00002

[3] Navi, B.B., Singer, S., Merkler, A.E., Cheng, N.T., Stone, J.B., Kamel, H., Iadecola, C., Elkind, M.S. and DeAngelis, L.M. (2014) Recurrent Thromboembolic Events after Ischemic Stroke in Patients with Cancer. Neurology, 83, 26-33. https://doi.org/10.1212/WNL.0000000000000539

[4] Navi, B.B. and Iadecola, C. (2018) Ischemic Stroke in Cancer Patients: A Review of an Underappreciated Pathology. Annals of Neurology, 83, 873-883. https://doi.org/10.1002/ana.25227

[5] Zaorsky, N.G., Zhang, Y., Tchelebi, L.T., Mackley, H.B., Chinchilli, V.M. and Zacharia, B.E. (2019) Stroke among Cancer Patients. Nature Communications, 10, 5172. https://doi.org/10.1038/s41467-019-13120-6

[6] Kim, S.J., Moon, G.J. and Bang, O.Y. (2013) Biomarkers for Stroke. Journal of Stroke, 15, 27-37. https://doi.org/10.5853/jos.2013.15.1.27

[7] Makris, K., Haliassos, A., Chondrogianni, M. and Tsivgoulis, G. (2018) Blood Biomarkers in Ischemic Stroke: Potential Role and Challenges in Clinical Practice and Research. Critical Reviews in Clinical Laboratory Sciences, 55, 294-328. https://doi.org/10.1080/10408363.2018.1461190

[8] Alvarez-Perez, F., Castelo-Branco, M. and Alvarez-Sabin, J. (2011) Usefulness of Measurement of Fibrinogen, D-Dimer, D-Dimer/Fibrinogen Ratio, C Reactive Protein and Erythrocyte Sedimentation Rate to Assess the Pathophysiology and Mechanism of Ischaemic Stroke. Journal of Neurology, Neurosurgery \& Psychiatry, 82, 986-992. https://doi.org/10.1136/jnnp.2010.230870

[9] Grisold, W., Oberndorfer, S. and Struhal, W. (2009) Stroke and Cancer: A Review. Acta Neurologica Scandinavica, 119, 1-16. https://doi.org/10.1111/j.1600-0404.2008.01059.x

[10] ten Wolde, M., Kraaijenhagen, R.A., Prins, M.H. and Büller, H.R. (2002) The Clinical Usefulness of D-Dimer Testing in Cancer Patients with Suspected Deep Venous Thrombosis. Archives of Internal Medicine, 162, 1880-1884. https://doi.org/10.1001/archinte.162.16.1880

[11] Seok, J.M., Kim, S.G., Kim, J.W., Chung, C.S., Kim, G.M., Lee, K.H. and Bang, O.Y. (2010) Coagulopathy and Embolic Signal in Cancer Patients with Ischemic Stroke. Annals of Neurology, 68, 213-219. https://doi.org/10.1002/ana.22050

[12] Kono, T., Ohtsuki, T., Hosomi, N., Takeda, I., Aoki, S., Sueda, Y., Ishihara, K., Nakamura, T., Yamawaki, T. and Matsumoto, M. (2012) Cancer-Associated Ischemic Stroke Is Associated with Elevated d-Dimer and Fibrin Degradation Product Levels in Acute Ischemic Stroke with Advanced Cancer. Geriatrics \& Gerontology International, 12, 468-474. https://doi.org/10.1111/j.1447-0594.2011.00796.x

[13] Bang, O.Y., Seok, J.M., Kim, S.G., Hong, J.M., Kim, H.Y., Lee, J., Chung, P.-W., Park, K.-Y., Kim, G.-M. and Chung, C.-S. (2011) Ischemic Stroke and Cancer: Stroke Severely Impacts Cancer Patients, While Cancer Increases the Number of Strokes. Journal of Clinical Neurology, 7, 53-59.

https://doi.org/10.3988/jen.2011.7.2.53

[14] Grobbee, D.E., Roest, M., Marx, J.J., Voorbij, H.A. and van der Schouw, Y.T. (2005) Serum Ferritin Is a Risk Factor for Stroke in Postmenopausal Women. Stroke, 36, 1637-1641. https://doi.org/10.1161/01.STR.0000173172.82880.72

[15] Wei, Y., Yang, Q., Qin, Q., Chen, Y., Quan, X., Wei, J., Zhou, L., Wang, D., Li, H. and Li, G. (2020) Profiling of the Risk Factors and Designing of a Model to Identify 
Ischemic Stroke in Patients with Non-Hodgkin Lymphoma: A Multicenter Retrospective Study. European Neurology, 83, 41-48. https://doi.org/10.1159/000506046

[16] Lyden, P. (2017) Using the National Institutes of Health Stroke Scale: A Cautionary Tale. Stroke, 48, 513-519. https://doi.org/10.1161/STROKEAHA.116.015434

[17] Lee, C.-H., Kang, H.G., Lee, J.S., Ryu, H.U. and Jeong, S.-K. (2018) Microbubble Signal and Trial of Org in Acute Stroke Treatment (TOAST) Classification in Ischemic Stroke. Journal of the Neurological Sciences, 390, 150-155. https://doi.org/10.1016/j.jns.2018.04.034

[18] Selvik, H.A., Thomassen, L., Bjerkreim, A.T. and Næss, H. (2015) Cancer-Associated Stroke: The Bergen NORSTROKE Study. Cerebrovascular Diseases Extra, 5, 107-113. https://doi.org/10.1159/000440730

[19] Sanossian, N., Djabiras, C., Mack, W.J. and Ovbiagele, B. (2013) Trends in Cancer Diagnoses among Inpatients Hospitalized with Stroke. Journal of Stroke and Cerebrovascular Diseases, 22, 1146-1150. https://doi.org/10.1016/j.jstrokecerebrovasdis.2012.11.016

[20] Zöller, B., Ji, J., Sundquist, J. and Sundquist, K. (2012) Risk of Haemorrhagic and Ischaemic Stroke in Patients with Cancer: A Nationwide Follow-Up Study from Sweden. European Journal of Cancer, 48, 1875-1883. https://doi.org/10.1016/j.ejca.2012.01.005

[21] Chaturvedi, S., Ansell, J. and Recht, L. (1994) Should Cerebral Ischemic Events in Cancer Patients Be Considered a Manifestation of Hypercoagulability? Stroke, 25, 1215-1218. https://doi.org/10.1161/01.STR.25.6.1215

[22] Cestari, D., Weine, D., Panageas, K., Segal, A. and DeAngelis, L. (2004) Stroke in Patients with Cancer: Incidence and Etiology. Neurology, 62, 2025-2030. https://doi.org/10.1212/01.WNL.0000129912.56486.2B

[23] Romeiro, A.C., Valadas, A. and Marques, J. (2015) Acute Ischemic Stroke on Cancer Patients, a Distinct Etiology? A Case-Control Study. Acta Medica Portuguesa, 28, 613-618. https://doi.org/10.20344/amp.6156

[24] Stefan, O., Vera, N., Otto, B., Heinz, L. and Wolfgang, G. (2009) Stroke in Cancer Patients: A Risk Factor Analysis. Journal of Neuro-Oncology, 94, 221-226. https://doi.org/10.1007/s11060-009-9818-3

[25] Kim, J.-M., Jung, K.-H., Park, K.H., Lee, S.-T., Chu, K. and Roh, J.-K. (2013) Clinical Manifestation of Cancer Related Stroke: Retrospective Case-Control Study. Journal of Neuro-Oncology, 111, 295-301. https://doi.org/10.1007/s11060-012-1011-4

[26] Sorgun, M.H., Kuzu, M., Ozer, I.S., Yilmaz, V., Ulukan, C., Levent, H.C., Tezcan, S., Rzayev, S., Rwanda, A. and Bakırarar, B. (2018) Risk Factors, Biomarkers, Etiology, Outcome and Prognosis of Ischemic Stroke in Cancer Patients. Asian Pacific Journal of Cancer Prevention: APJCP, 19, 649.

[27] Zhang, Y.Y., Chan, D.K.Y., Cordato, D., Shen, Q. and Sheng, A.Z. (2006) Stroke Risk Factor, Pattern and Outcome in Patients with Cancer. Acta Neurologica Scandinavica, 114, 378-383. https://doi.org/10.1111/j.1600-0404.2006.00709.x

[28] Schwarzbach, C.J., Schaefer, A., Ebert, A., Held, V., Bolognese, M., Kablau, M., Hennerici, M.G. and Fatar, M. (2012) Stroke and Cancer: The Importance of Cancer-Associated Hypercoagulation as a Possible Stroke Etiology. Stroke, 43, 3029-3034. https://doi.org/10.1161/STROKEAHA.112.658625

[29] Kim, S.G., Hong, J.M., Kim, H.Y., Lee, J., Chung, P.-W., Park, K.-Y., Kim, G.-M., Lee, K.H., Chung, C.-S. and Bang, O.Y. (2010) Ischemic Stroke in Cancer Patients 
with and without Conventional Mechanisms: A Multicenter Study in Korea. Stroke, 41, 798-801. https://doi.org/10.1161/STROKEAHA.109.571356

[30] Kalantar-Zadeh, K., Rodriguez, R.A. and Humphreys, M.H. (2004) Association between Serum Ferritin and Measures of Inflammation, Nutrition and Iron in Haemodialysis Patients. Nephrology Dialysis Transplantation, 19, 141-149.

https://doi.org/10.1093/ndt/gfg493

[31] Foley, J.H. and Conway, E.M. (2016) Cross Talk Pathways between Coagulation and Inflammation. Circulation Research, 118, 1392-1408. https://doi.org/10.1161/CIRCRESAHA.116.306853

[32] Kernan, K.F. and Carcillo, J.A. (2017) Hyperferritinemia and Inflammation. International Immunology, 29, 401-409. https://doi.org/10.1093/intimm/dxx031

[33] Dardiotis, E., Alonzo, A.-M., Markoula, S., Siokas, V., Tsarouhas, K., Tzanakakis, G., Libra, M., Kyritsis, A.P., British, A.G. and Aschner, M. (2019) Cancer-Associated Stroke: Pathophysiology, Detection and Management. International Journal of Oncology, 54, 779-796. https://doi.org/10.3892/ijo.2019.4669

[34] Navi, B.B., Reiner, A.S., Kamel, H., Iadecola, C., Elkind, M.S., Panageas, K.S. and DeAngelis, L.M. (2015) Association between Incident Cancer and Subsequent Stroke. Annals of Neurology, 77, 291-300. https://doi.org/10.1002/ana.24325 